\title{
Montelukast inhibits RANKL-induced osteoclast formation and bone loss via CysLTR1 and P2Y12
}

\author{
JU-HEE KANG ${ }^{1}$, HYUNGSIK LIM ${ }^{2}$, DONG-SEOK LEE ${ }^{3}$ and MIJUNG YIM ${ }^{1}$
}

\begin{abstract}
${ }^{1}$ College of Pharmacy and Research Institute of Pharmaceutical Sciences, Sookmyung Women's University, Seoul 140-742, Republic of Korea; ${ }^{2}$ Department of Physics, Hunter College of The City University of New York, New York, NY 10065, USA; ${ }^{3}$ Department of Natural Sciences, School of Life Sciences, BK21 Plus KNU Creative BioResearch Group, College of Natural Sciences, Kyungpook National University, Daegu 41566, Republic of Korea
\end{abstract}

Received November 16, 2017; Accepted April 17, 2018

DOI: $10.3892 / \mathrm{mmr} .2018 .9179$

\begin{abstract}
Osteoclasts (OCs) are resorptive cells responsible for bone erosion in diseases, including osteoporosis, periodontitis and rheumatoid arthritis. Montelukast is a cysteinyl leukotriene receptor 1 (CysLTR1) antagonist clinically used for the treatment of asthma. In the present study, the role of CysLTR1 on OC formation and bone loss was investigated using montelukast. Montelukast inhibited receptor activator of nuclear factor- $\kappa \mathrm{B}$ ligand (RANKL)-induced OC formation in cultures of mouse bone marrow macrophages. Additionally, montelukast suppressed actin ring formation and bone resorption activity of differentiated OCs. The inhibitory effect of montelukast was associated with impaired activation of extracellular signal-regulated kinase, AKT serine/threonine kinase, and/or phospholipase $\mathrm{C} \gamma 2$ signaling pathways downstream of RANK, followed by decreased expression of nuclear factor of activated $\mathrm{T}$ cells c1. Notably, OC formation was efficiently restored by addition of adenosine diphosphate, a P2Y12 agonist, as well as by addition of CysLT. Furthermore, similar to montelukast, P2Y12 blockade by a pharmacological inhibitor or siRNAs suppressed OC differentiation. These data indicate the involvement of the $\mathrm{P} 2 \mathrm{Y} 12$ receptor in the inhibitory effect of montelukast on osteoclastogenesis. In vivo, montelukast significantly inhibited inflammation-induced osteoclastogenesis in the calvarial model. Montelukast also served a protective role in a murine ovariectomy (OVX)- and unloading-induced bone loss model. Altogether, these results confirmed that the CysLTR1 antagonist exerted an inhibitory effect on OC formation in vitro and in vivo. It may be useful
\end{abstract}

Correspondence to: Professor Mijung Yim, College of Pharmacy and Research Institute of Pharmaceutical Sciences, Sookmyung Women's University, 52 Hyochangwongil, Yongsan, Seoul 140-742, Republic of Korea

E-mail: myim@sm.ac.kr

Key words: montelukast, cysteinyl leukotriene receptor 1 antagonist, P2Y12, osteoclast, nuclear factor- $\kappa \mathrm{B}$ ligand, nuclear factor of activated $\mathrm{T}$ cells 1 , bone loss for the treatment of bone diseases associated with excessive bone resorption.

\section{Introduction}

Bone homeostasis is tightly regulated by the balance between osteoblasts (OBs), the bone-forming cells, and osteoclasts (OCs), the bone-resorbing cells (1). Pathological conditions such as osteoporosis, inflammation, and cancer break this balance in favor of the augmentation of OC differentiation and activity, leading to net bone loss (1). As a result, patients with these diseases suffer from increased risk of bone fracture. Therefore, uncovering potential therapeutic targets by understanding $\mathrm{OC}$ differentiation and function are important objectives to treat these diseases (2).

OC differentiation is regulated by macrophage colony-stimulating factor (M-CSF) and receptor activator of nuclear factor- $\kappa \mathrm{B}$ ligand (RANKL) $(3,4)$. M-CSF is crucial for myeloid cell survival and differentiation to pre-osteoclasts, and RANKL elicits pre-osteoclast fusion, polykaryon maturation, and $\mathrm{OC}$ activation. Mechanistically, the binding of RANKL to its cell surface receptor, RANK, generates the activation of multiple downstream pathways such as the nuclear factor- $\kappa \mathrm{B}(\mathrm{NF}-\kappa \mathrm{B})$, mitogen-activated protein kinases (MAPKs), phosphatidylinositol 3-kinase/Akt (PI3K/Akt), and phospholipase $\mathrm{C}$ gamma 2 (PLC $\gamma 2$ )- $\mathrm{Ca}^{2+}$-CREB signaling pathways (5-7), which leads to subsequent nuclear translocation of nuclear factor of activated T cells, cytoplasmic 1 (NFATc1), a master transcription factor for osteoclastogenesis (8-10).

Cysteinyl leukotrienes (CysLTs) are potent lipid mediators of several immune and inflammatory stimuli (11). Three different CysLTs $\left(\mathrm{LTC}_{4}, \mathrm{LTD}_{4}\right.$, and $\left.\mathrm{LTE}_{4}\right)$ arise from a single intracellular synthetic event by successive enzymatic conversions. Arachidonic acid is oxidized by 5-lipoxygenase (5-LO) to generate the unstable precursor leukotriene $\mathrm{A}_{4}\left(\mathrm{LTA}_{4}\right)$, and subsequently, leukotriene $\mathrm{C}_{4}$ synthase $\left(\mathrm{LTC}_{4} \mathrm{~S}\right)$ converts $\mathrm{LTA}_{4}$ to the parent CysLT, $\mathrm{LTC}_{4}$. After energy-dependent export from the cell, $\mathrm{LTC}_{4}$ is sequentially converted to $\mathrm{LTD}_{4}$, and

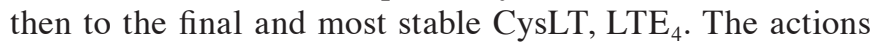
of CysLTs are mediated via at least two types of CysLT receptors, designated as CysLT receptor 1 (CysLTR1) and 2 (CysLTR2). CysLTs-CysLTR1 signaling is considered a major 
pharmacological target for the treatment of asthma and allergic rhinitis $(11,12)$. Several CysLTR1 antagonists such as montelukast have been approved by the Food and Drug Agency (FDA) and are in the market for the treatment of these diseases (12). Recently, expanded roles of this signaling pathway in the pathogenesis of cardiovascular diseases, cerebrovascular diseases, malignant tumors, fibrosis, and immune host defense have been suggested $(11,13)$, thereby indicating that it could be involved in various pathophysiological conditions as well as in inflammation in a tissue-specific manner.

Although several studies reported the involvement of leukotriene in OC differentiation and function (14), little is known about the effect of CysLTR1 on OCs and bone loss. Thus, we explored the detailed mechanism by which CysLTR1 regulates OC formation and its protective effect on bone loss using CysLTR1 antagonist to re-position the CysLTR1 as a promising drug target in bone-related diseases in association with excessive osteoclastogenesis.

\section{Materials and methods}

Reagents. CysLTR1 antagonist (Montelukast) and leukotriene $\mathrm{C}_{4}$ were purchased from Cayman Chemical Company, (Ann Arbor, MI, USA). P2Y12 antagonist (MRS2395) and 2-(Methylthio)-ADP were purchased from Santa Cruz Biotechnology, Inc. (Dallas, TX, USA). Antibodies against phospho-PLC 2 , phospho-Akt, phospho-ERK, ERK, and c-Fos were purchased fromCell Signaling Technology, Inc., (Danvers, MA, USA). Antibody against NFATc1 was purchased from Santa Cruz Biotechnology, Inc. Antibody against phospho-CREB was purchased from EMD Millipore (Billerica, MA, USA). Antibody against $\beta$-actin was purchased from Sigma-Aldrich; Merck KGaA (Darmstadt, Germany). All other reagents were from Sigma-Aldrich; Merck KGaA.

Animals. Male ICR (4- to 6-week-old) mice were purchased from Samtako Inc., (Osan, Korea). Female C57BL/6J (6- to 7-week-old) mice were purchased from DBL Inc., (Umsung, Korea). All mice were maintained in the animal facility of the Sookmyung Women's University in a 12:12 h light-dark cycle at $21 \pm 2^{\circ} \mathrm{C}$, and were allowed food and water ad libitum. All experiments were performed in accordance with institutional guidelines approved by the Sookmyung Women's University Care and Use Committee.

OC differentiation. Mouse bone marrow cells were obtained from the long bones of 4-to 6-week-old male mice. Bone marrow cells were cultured in $\alpha$-MEM (10\% FBS), supplemented with M-CSF (10 ng/ml; R\&D Systems, Inc., Minneapolis, MN, USA) for $12 \mathrm{~h}$ to separate adherent and non-adherent cells. The non-adherent cells were then harvested and cultured with M-CSF (30 ng/ml). After 3 days of culture, the floating cells were removed and the attached cells used as bone marrow-derived macrophages (BMMs). BMMs (1x10 $)$ were plated in 96-well plates and differentiated into OCs by further treatment with M-CSF (30 ng/ml) and RANKL (100 ng/ml). Cells were then fixed and permeabilized with an equal volume mixture of acetone and ethanol for $30 \mathrm{~s}$ and then treated with tartrate resistant acid phosphatase (TRAP) staining solution
(0.01\% naphthol AS-MX phosphate (Sigma-Aldrich; Merck $\mathrm{KGaA}$ ) and 0.06\% Fast Red Violet LB salt (Sigma-Aldrich; Merck KGaA) in $50 \mathrm{mM}$ sodium tartrate dehydrate and $45 \mathrm{mM}$ sodium acetate ( $\mathrm{pH}$ 5.0)). TRAP-positive multinucleated cells (>3 nuclei/cell) were counted as mature osteoclasts.

Reverse transcription-quantitative polymerase chain reaction (RT-qPCR) analysis. Total RNA was purified with easy-BLUE (iNtRON Biotechnology, Seoul, Korea), and cDNA was prepared from $5 \mu \mathrm{g}$ of RNA using Revert Aid ${ }^{\mathrm{TM}}$ First-Strand cDNA Synthesis kit (Fermentas; Thermo Fisher Scientific, Inc., Waltham, MA, USA). RT-qPCR reactions were performed in a total volume of $20 \mu \mathrm{l}$ using SYBR-Green PCR Master mix (Applied Biosystems; Thermo Fisher Scientific, Inc.) according to the manufacturer's protocol. Thermocycling was performed using a 7500 real-time PCR System (Applied Biosystems; Thermo Fisher Scientific, Inc.) with the following conditions: initial hold at $95^{\circ} \mathrm{C}$ for $10 \mathrm{~min}$, followed by 40 cycles of denaturation at $95^{\circ} \mathrm{C}$ for $15 \mathrm{sec}$, annealing at $58^{\circ} \mathrm{C}$, and extension at $60^{\circ} \mathrm{C}$ for $1 \mathrm{~min}$. Specific primer sequences for RT-qPCR were designed as follows: calcitonin receptor (CTR), 5'-tttcaagaaccttagctgccagag-3' (forward), 5'-caaggcacggacaat gttgagaag-3' (reverse); cathepsin K, 5'-cttccaatacgtgcagcaga-3' (forward), 5'-acgcaccaatatcttgcacc-3' (reverse); Atp6v0d2, 5'-tcagatctcttcaaggctgtgctg-3' (forward), 5'-gtgccaaatgagttcaga gtgatg-3' (reverse); DC-STAMP, 5'-tggaagttcacttgaaactacgtg-3' (forward), 5'-ctcggtttcccgtcagcctctctc-3' (reverse); $\alpha \mathrm{v}$-integrin, 5'-cctcagagagggagatgttcacac-3' (forward), 5'-aactgccaagatgat cacccacac-3' (reverse); $\beta 3$-integrin, 5'-gatgacatcgagcaggtg aaagag-3' (forward), 5'-ccggtcatgaatggtgatgagtag-3' (reverse); GAPDH, 5'-tgcaccaccaactgcttagc-3' (forward), 5'-ggcatggactgt ggtcatgag-3' (reverse). Data were analyzed using 7500 System Sequence Detection Software v2.0 (Applied Biosystems; Thermo Fisher Scientific, Inc.). An index mRNA level was assessed using a threshold cycle $(\mathrm{Cq})$ value and normalized against GAPDH expression. qPCR results were calculated using the $2^{-\Delta \Delta \mathrm{Cq}}$ method (15).

Western blot analysis. Total cell lysates were separated by SDS-PAGE and transferred onto Immobilon-P membranes (EMD Millipore). The membranes were blocked with $5 \%$ non-fat-milk in PBS-T, and then immunostained with anti-phospho-PLC $\gamma 2$ (1:1,000), anti-phospho-Akt $(1: 1,000)$, anti-phospho CREB (1:1,000), anti-phospho ERK $(1: 1,000)$, anti-ERK $(1: 1,000)$, anti-c-Fos $(1: 1,000)$, anti-NFATc1 $(1: 200)$, or anti- $\beta$-actin $(1: 4,000)$, followed by secondary horseradish peroxidase-conjugated antibody $(1: 5,000)$. The membranes were developed using an enhanced chemiluminescence detection kit (GE Healthcare Life Sciences, Little Chalfont, UK).

Actin ring staining. Cells were washed twice with $\mathrm{PBS}$ and fixed with $10 \%$ formalin for $5 \mathrm{~min}$. Cells were permeabilized with $\mathrm{EtOH} /$ Acetone $(1: 1)$ for $1 \mathrm{~min}$ at room temperature, the solvent removed, and the cells dried. Actin rings were stained with rhodamine-conjugated phalloidin (Molecular Probes; Thermo Fisher Scientific, Inc.) overnight in PBS at $4^{\circ} \mathrm{C}$ in the dark. After washing the cells twice with PBS, the number of actin rings was counted using a fluorescence microscope (Olympus Corporation, Tokyo, Japan). 
Bone resorption assay. BMMs were differentiated on dentin slices with M-CSF and RANKL (PeproTech, Inc., Rocky Hill, NJ, USA) for 3 days, and treated with montelukast or MRS2395 for 4 days. The cells were removed from the dentin slice by wiping the surface, and then the slices were stained with toluidine blue ( $1 \mu \mathrm{g} / \mathrm{ml}$; J.T. Baker Chemical Co., Phillipsburg, NJ, USA). The number of pits formed by bone resorption on the dentin slices was counted.

Retroviral gene transduction. To generate retroviral stocks, PMX-puro GFP (GFP-vector) or PMSCV-GFP NFATc1 (NFATc1) was transfected into the packaging cell line platinum-E (Plat-E). Viral supernatant was collected from culture media at $48 \mathrm{~h}$ after transfection using Lipofectamine 2000 (Invitrogen; Thermo Fisher Scientific, Inc.) according to the manufacturer's instructions. For infection with retroviruses, BMMs were incubated with the viral supernatant (4 ml/dish), polybrene (10 $\mu \mathrm{g} / \mathrm{ml}$, Santa Cruz Biotechnology, Inc.), and M-CSF (30 ng/ml) for 2 days, and selected by puromycin (2 $\mu \mathrm{g} / \mathrm{ml}$, Sigma-Aldrich) for an additional $48 \mathrm{~h}$.

siRNA transfection. BMMs were plated on 48-well plates at a density of $3.5 \times 10^{4}$ cells/well with $30 \mathrm{ng} / \mathrm{ml} \mathrm{M-CSF}$. After $24 \mathrm{~h}$, cells were transfected with $40 \mathrm{nM}$ mouse P2Y12 on-target siRNAs (sc-151960; Santa Cruz Biotechnology, Inc.) using Lipofectamine 2000 (Invitrogen; Thermo Fisher Scientific, Inc.) according to the manufacturer's instructions. The control contained $40 \mathrm{nM}$ non-targeting siRNA (Qiagen $\mathrm{GmbH}$, Hilden, Germany). The transfection was performed in $2.5 \mathrm{ml}$ of serum free medium for $6 \mathrm{~h}$; the cells were then cultured for 3 days in complete medium containing $30 \mathrm{ng} / \mathrm{ml} \mathrm{M-CSF}$ and $100 \mathrm{ng} / \mathrm{ml}$ RANKL to form osteoclasts.

Lipopolysaccharide (LPS)-induced osteoclast formation in vivo. ICR mice (5-week-old) were i.p. injected with vehicle $(\mathrm{EtOH})$ or montelukast $(5 \mathrm{mg} / \mathrm{kg})$ every day. The day after the first injection of montelukast, LPS $(0.5 \mathrm{mg})$ or PBS was directly injected on the calvarium. Six days after LPS injection, mice were euthanized and the calvaria were extracted ( $n=5$ per each group). Calvaria were fixed in $4 \%$ paraformaldehyde for $24 \mathrm{~h}$ at $4^{\circ} \mathrm{C}$, and then stained for TRAP. Image analysis was performed by using ImageJ software (v1.32; National Institutes of Health, Bethesda, MD, USA) according to the manufacturer's protocol.

Ovariectomy $(O V X)$-induced bone loss in vivo. Female mice (9-week-old, C57BL/6J) were used for ovariectomy. After 5 days, vehicle (1\% methylcellulose) or montelukast $(5 \mathrm{mg} / \mathrm{kg})$ was orally administered daily for 12 weeks ( $n=7$ per each group). Femurs were extracted and stored in $70 \%$ ethanol at $4^{\circ} \mathrm{C}$ until analysis. Two-dimensional measurement was performed with a micro-CT scanner and associated analysis software (Model 1172; Bruker microCT, Kontich, Belgium) at 9- $\mu \mathrm{m}$ voxel size. Image acquisition was performed at $35 \mathrm{kV}$ of energy and $220 \mathrm{~mA}$ of intensity (590 ms of integration time). The threshold was set to segment the bone from the background, and the same threshold setting was used for all samples.

Hind limb unloading model. Eight-week-old male ICR mice were orally administered vehicle (1\% methylcellulose) or montelukast $(5 \mathrm{mg} / \mathrm{kg}$ ) daily. After a day, mice were subjected to hind limb unloading for 14 days. Hind limb unloading was conducted by applying a tape to the surface of the hind limb to set a metal clip (16). The other end of the clip was fixed to an overhead bar. The height of the bar was adjusted to maintain the mice at $30^{\circ}$ head down tilt with the hind limbs elevated above the floor of the cage. Loaded control mice were also housed under the same conditions except for hind limb unloading for the same duration ( $n=7$ per each group). Femurs were extracted and stored in $70 \%$ ethanol at $4{ }^{\circ} \mathrm{C}$ and analyzed by micro-CT as described above.

Statistical analysis. Data are presented as the mean \pm standard deviation from at least three independent experiments. The statistical significance of differences between groups was determined using Student's t-test and analysis of variance with a least significant difference post hoc test. $\mathrm{P}<0.05$ was considered to indicate a statistically significant difference.

\section{Results}

CysLTR1 antagonist inhibits RANKL-induced OC formation and bone resorption in vitro. RANKL is essential and sufficient for the differentiation of OC precursors into mature OCs in the presence of M-CSF $(3,4)$. Thus, we examined the effects of CysLTR1 antagonist on RANKL-induced OC formation from bone-marrow-derived macrophages (BMMs). We used montelukast since it is a commonly prescribed CysLTR1 antagonist and is generally considered a safe drug with the occurrence of few adverse drug reactions (17). When BMMs were incubated with M-CSF and RANKL for 4 days, numerous TRAP-positive multinucleated OCs were generated. Treatment of the same cultures with montelukast completely suppressed OC formation (Fig. 1A). Consistently, the mRNA expression levels of various OC markers such as calcitonin receptor (CTR), cathepsin K, Atp6v0d2, DC-STAMP, $\alpha$-integrin, and $\beta 3$-integrin were increased in cultures treated with M-CSF and RANKL for 4 days. This effect was dramatically suppressed by montelukast treatment (Fig. 1B).

Since montelukast is known to block the effects of CysLTs by competitive binding to CysLTR1 (11), we next investigated whether addition of CysLT rescues the inhibitory effect of montelukast on OC formation. Suppression of osteoclastogenesis by montelukast was partly rescued by addition of exogenous $\mathrm{LTC}_{4}$, suggesting the partial involvement of CysLTR1 in the inhibition of OC formation by montelukast (Fig. 1C).

We next investigated the effect of montelukast on mature OCs. Mature OCs exhibit highly polarized morphological features, which is an essential event to initiate bone resorption (18). When mature OCs were incubated with RANKL, a clear cytoplasm and a smooth periphery were observed in polarized OCs. Conversely, the presence of montelukast induced morphological changes such as a contracted cytoplasm and an irregular cell periphery within $24 \mathrm{~h}$ (Fig. 1D). When we further observed the morphology of OCs after removal of montelukast from the culture medium, the number of mature OCs with a clear cytoplasm and a smooth periphery increased along with the removal of montelukast (Fig. 1D). Since actin rings are believed to be one of the markers of polarized OCs (3), 

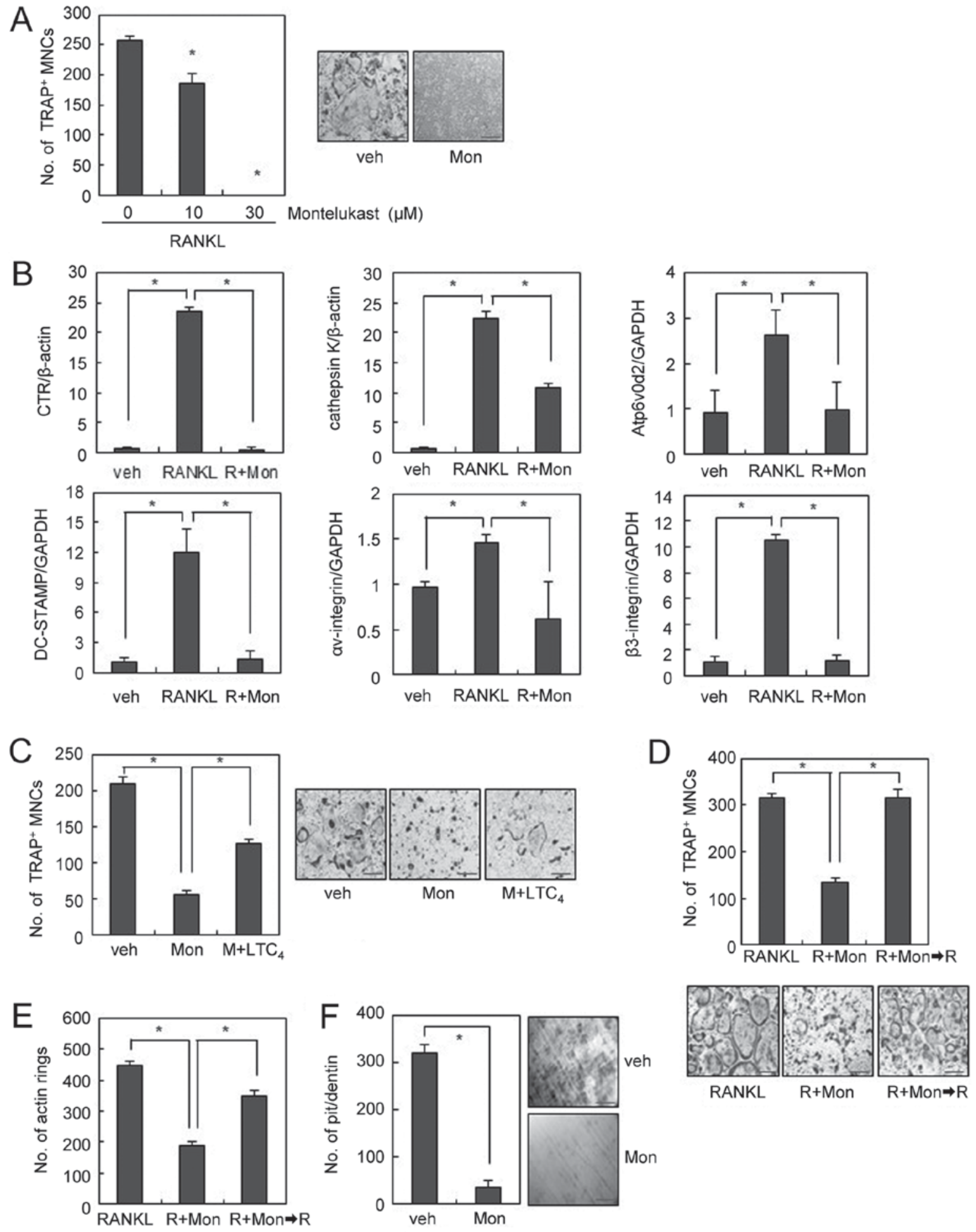

Figure 1. Montelukast inhibits RANKL-induced OC formation and bone resorption. (A) BMMs were cultured with M-CSF (30 ng/ml) and RANKL (100 ng/ml) in the absence or presence of montelukast for 4 days and stained for TRAP activity. TRAP-positive cells that had $>3$ nuclei were counted as OCs. (B) mRNA expression was determined by reverse transcription-quantitative polymerase chain reaction. (C) BMMs were cultured with montelukast (30 $\mu \mathrm{M})$ in the absence or presence of leukotriene $\mathrm{C}_{4}(50 \mathrm{nM})$ during osteoclast differentiation and stained for TRAP activity. (D and E) Mature OCs were treated with montelukast $(30 \mu \mathrm{M})$. Following $24 \mathrm{~h}$, montelukast was removed from the culture medium and mature OCs were cultured for $24 \mathrm{~h}$. (D) Actin rings or (E) OCs were stained with rhodamine-conjugated phalloidin or TRAP staining solution. (F) BMMs were differentiated on dentine slices with M-CSF (30 ng/ml) and RANKL $(100 \mathrm{ng} / \mathrm{ml})$ for 3 days, and treated with montelukast $(30 \mu \mathrm{M})$ for 4 days. The resorbed pit numbers were counted. Scale bars=200 $\mu \mathrm{m}$. Data are expressed as mean \pm standard deviation from at least three independent experiments. ${ }^{*} \mathrm{P}<0.05$, as indicated. RANKL, receptor activator of nuclear factor- $\kappa \mathrm{B}$ ligand; OCs, osteoclasts; BMM, bone marrow-derived macrophages; TRAP, tartrate resistant acid phosphatase; veh, vehicle; R, RANKL; Mon, montelukast.

we examined whether the effect of montelukast on actin ring formation was involved in the morphological changes of OCs. F-actin staining showed that montelukast efficiently disrupted the formation of actin rings in mature OCs, which was recovered after removal of montelukast (Fig. 1E). These data suggest that montelukast reversibly induces the disruption 
of actin rings, causing reversible morphological changes of mature OCs. To investigate whether the effect of montelukast on the morphology of OCs could be reflected in the osteoclastic activity, we performed an in vitro resorption pit assay using dentine slices. Many resorption pits were generated by RANKL-treated OCs (Fig. 1F). In contrast, montelukast treatment strongly inhibited the formation of resorption pits by RANKL-treated OCs (Fig. 1F). Together, these results suggest that the CysLTR1 antagonist exerts inhibitory effects on the morphology of OCs, which leads to reduced bone resorption.

CysLTR1 antagonist regulates osteoclastogenesis through NFATc1. The NFATc1 pathway plays a critical and fundamental role in OC development and the lack of NFATc1 arrests osteoclastogenesis (8). Thus, we investigated the effect of montelukast on the NFATc1 pathway. Montelukast abolished RANKL-induced NFATc1 expression in BMMs (Fig. 2A). We next investigated whether the effect of montelukast was rescued by the overexpression of NFATc1. The NFATc1-transduced BMMs were cultured with M-CSF and RANKL in the absence or presence of montelukast for 3 days. As shown in Fig. 2B, suppression of osteoclastogenesis by montelukast was efficiently overcome by the forced expression of NFATcl, suggesting that the anti-osteoclastogenic effect of montelukast is mainly due to a reduction in NFATc1 expression.

To identify the molecular mechanism underlying the inhibitory effects of montelukast on osteoclastogenesis, we next examined the effects of montelukast on early signaling pathways such as ERK, Akt, and/or phospholipase $\mathrm{C} \gamma 2$ (PLC $\gamma 2$ ), which activate the NFATc1 pathway (5-7).

As shown in Fig. 2C-E, phosphorylation of ERK, Akt, and PLC $\gamma 2$ was observed $15 \mathrm{~min}$ after RANKL treatment, but suppressed by pretreatment with montelukast. PLC $\gamma 2$ phosphorylation increases intracellular calcium levels, which in turn activates calcium-dependent transcriptional factors such as cAMP response element-binding protein $(\mathrm{CREB})(7,19)$. Since CREB activation plays an essential role in NFATc1 induction during osteoclastogenesis $(9,10)$, we further examined whether montelukast inhibits CREB activation. RANKL stimulation led to CREB phosphorylation, and montelukast interfered with this process (Fig. 2F). Taken together, these data suggest that montelukast regulates NFATc1 via ERK, Akt, and PLC $\gamma 2$ signaling pathways.

CysLTR1 antagonist regulates osteoclastogenesis via CysLTR1 and P2Y12. Since the inhibition of osteoclastogenesis by montelukast was only partially rescued by $\mathrm{LTC}_{4}$ (Fig. 1C), we speculated that the inhibitory effect of montelukast on OC formation is likely to involve mechanisms other than CysLTR1 blockage. In an effort to identify an alternative receptor for montelukast, we found that the addition of adenosine diphosphate (ADP), a P2Y12 agonist, also rescued the blockade of OC formation by montelukast (Fig. 3A). These data suggest that the inhibitory effect of montelukast on osteoclastogenesis was partly due to its suppressive actions on $\mathrm{P} 2 \mathrm{Y} 12$ receptor. To confirm the regulatory role of P2Y12 on OC formation, we next investigated the involvement P2Y12 receptor in OC differentiation. MRS2395, a P2Y12 antagonist, decreased the formation of TRAP-positive OCs in a dose-dependent manner (Fig. 3B). The suppression of osteoclastogenesis by MRS2395 was successfully rescued by addition of exogenous ADP, suggesting the involvement of P2Y12 in the inhibition of OC formation by MRS2395 (Fig. 3C). Similar inhibitory effects on osteoclastogenesis were also obtained by using P2Y12-specific siRNAs (Fig. 3D). Accordingly, the RANKL-induced expression of various OC markers was suppressed by MRS2395 treatment (Fig. 3E). Furthermore, MRS2395 significantly inhibited the resorption pit formation activity of OCs in dentine slices (Fig. 3F).

We next examined the effect of P2Y12 blockade on RANKL-induced NFATc1 expression. Pharmacological P2Y12 inhibition by MRS2395 or down-regulation of P2Y12 by siRNA decreased RANKL-induced NFATc1 expression (Fig. 4A and B). In addition, suppression of osteoclastogenesis by MRS2395 was successfully overcome by the forced expression of NFATc1 (Fig. 4C). MRS2395 abolished RANKL-induced activation of ERK and Akt, as montelukast did (Fig. 4D and E). Taken together, these data suggest that P2Y12 is involved in OC differentiation, and the inhibitory effect of montelukast on OC formation is partly due to P2Y12 blockade.

CysLTR1 antagonist prevented ovariectomy-or unloading-induced bone loss in vivo. LPS is produced by gram-negative bacteria and induces the secretion of TNF and other inflammatory cytokines, leading to systemic inflammatory response. Injection of LPS in animals can mimic OC-mediated bone destruction that occurs under sepsis (20). To further investigate the anti-osteoclastogenic effect of the CysLTR1 antagonist in vivo, we injected LPS into the supracalvarial region of mice with or without montelukast. TRAP staining of whole calvariae showed that LPS dramatically increased OC numbers (Fig. 5A and B). In parallel with its anti-osteoclastogenic effect in vitro, montelukast notably reduced LPS-induced OC formation (Fig. 5A and B). These data suggest that the CysLTR1 antagonist has an inhibitory effect on inflammation-induced osteoclastogenesis in vivo.

To further explore the therapeutic effects of montelukast on pathological bone loss, we next used the ovariectomized (OVX) or hind limb unloading mouse model to mimic menopause- or disuse-induced osteoporosis. The $\mu \mathrm{CT}$ analysis demonstrated that OVX significantly decreased the values of bone mineral density (BMD), bone volume/tissue volume (BV/TV), trabecular number (Tb.N), and trabecular thickness (Tb.Th), and increased the values of trabecular separation (Tb.Sp) compared with the sham operation. In contrast, in OVX mice, montelukast treatment significantly inhibited the OVX-induced bone loss assessed by measuring these parameters (Fig. 5C). In addition, the higher structure model index (SMI) number, an indicator of increased fragility, in OVX mice was significantly decreased by montelukast treatment (Fig. 5C). Similar results were demonstrated by a two-dimensional visualization of the femoral area (Fig. 5D). Furthermore, $\mu \mathrm{CT}$ analysis for cortical bone confirmed that OVX significantly decreased the values of cortical area $(\mathrm{Ct}$. Ar), cortical thickness (Ct.TH), and BMD compared with the sham operation (Fig. 5E). By treating montelukast, OVX-induced cortical bone loss was suppressed (Fig. 5E). Montelukast also showed the therapeutic effects in disuse 
A
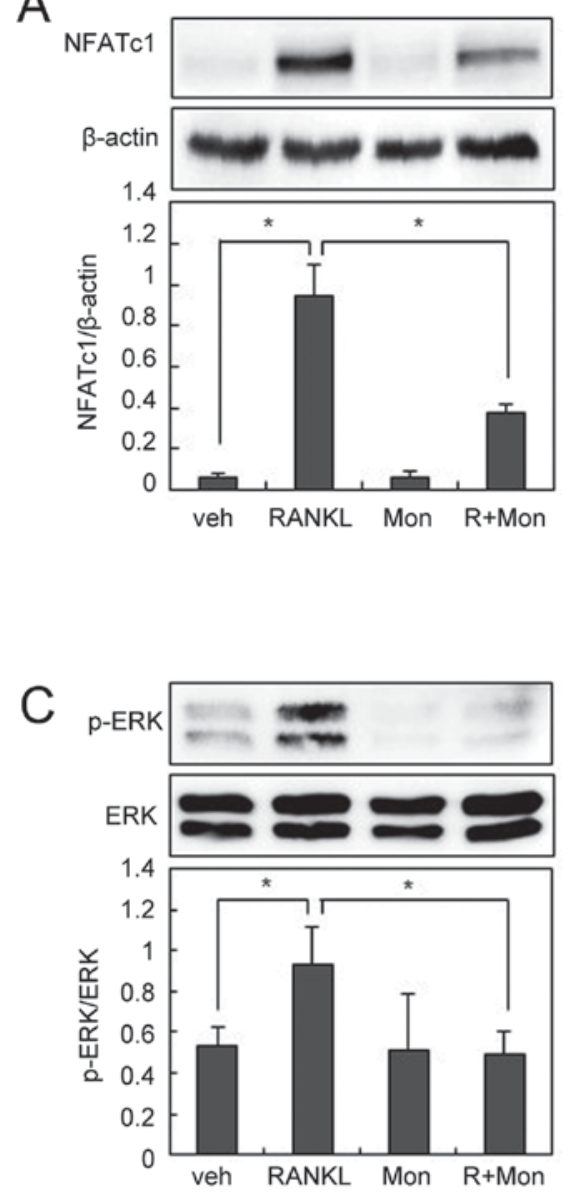

$\mathrm{E}$

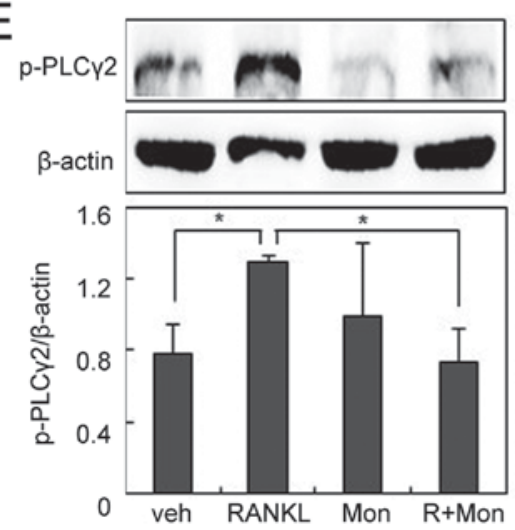

B
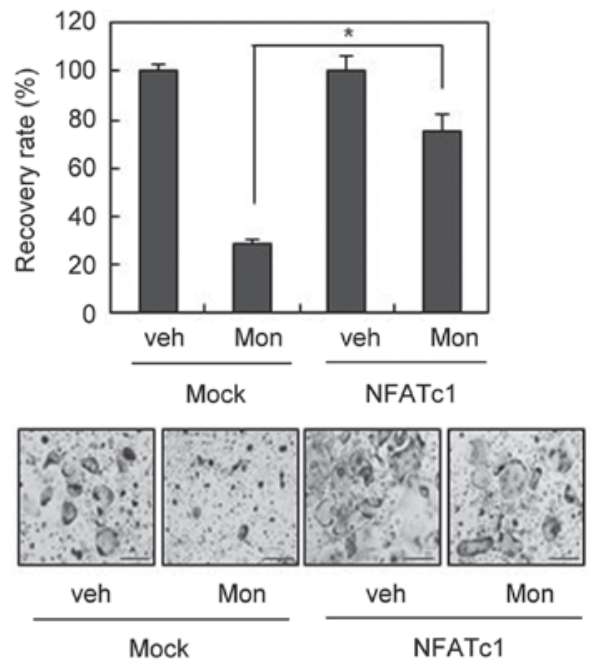

D

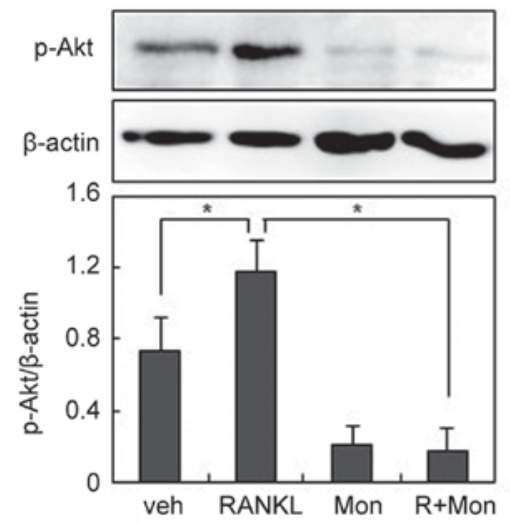

$\mathrm{F}$

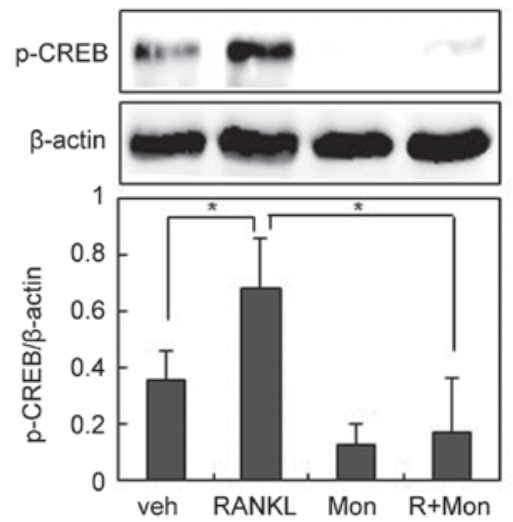

Figure 2. Montelukast suppresses RANKL-induced NFATc1 expression. (A) BMMs were pre-incubated in the absence or presence of montelukast (30 $\mu \mathrm{M}$ ) for $30 \mathrm{~min}$ and then cultured with or without RANKL (200 ng/ml) for $24 \mathrm{~h}$. Cell lysates were subjected to western blotting analysis. (B) BMMs were infected through a retrovirus packaging system. Infected BMMs were cultured with RANKL (100 ng/ml) and macrophage colony-stimulating factor (30 ng/ml) in the presence or absence of montelukast $(30 \mu \mathrm{M})$ for 3 days. TRAP-positive cells that had $>3$ nuclei were counted. The recovery rate was defined as the percentage of OC formation in the presence of montelukast. BMMs were pre-incubated in the absence or presence of montelukast (30 $\mu \mathrm{M})$ for 30 min, and then treated with or without $200 \mathrm{ng} / \mathrm{ml}$ of RANKL for $15 \mathrm{~min}$ (p-ERK, p-Akt, and p-PLC $\gamma 2$ ) or $3 \mathrm{~h}$ (p-CREB). Cell lysates were then subjected to western blot analysis for (C) p-ERK and ERK, (D) p-Akt, (E) p- PLC $\gamma 2$ and (F) p-CREB. Scale bars=200 $\mu \mathrm{m}$. Data are expressed as means standard deviation from at least three independent experiments. " $\mathrm{P}<0.05$, as indicated. RANKL, receptor activator of nuclear factor- $\mathrm{KB}$ ligand; OCs, osteoclasts; BMM, bone marrow-derived macrophages; TRAP, tartrate resistant acid phosphatase; NFATc1, nuclear factor of activated T cells, cytoplasmic 1; p-, phosphorylated; ERK, extracellular signal-regulated kinase; Akt, protein kinase B; PLC $\gamma 2$, phospholipase $\mathrm{C} \gamma 2$; CREB, cyclic adenosine monophosphate response element-binding protein; veh, vehicle; R, RANKL; Mon, montelukast.

osteoporosis. Two weeks after hind limb unloading, the bone loss was less severe in montelukast-treated mice than that in the vehicle-treated mice (Fig. 6A). Montelukast-treated mice showed higher BMD compared to vehicle-treated mice (Fig. 6B). In addition, unloading-induced structural bone alterations, including decreases in BV/TV, Tb.Th, and Tb.N and increases in Tb.Sp and SMI were abolished by montelukast treatment (Fig. 6B). Furthermore, $\mu \mathrm{CT}$ analysis for cortical bone confirmed that unloading condition significantly decreased the values of cortical area (Ct.Ar), cortical 
A
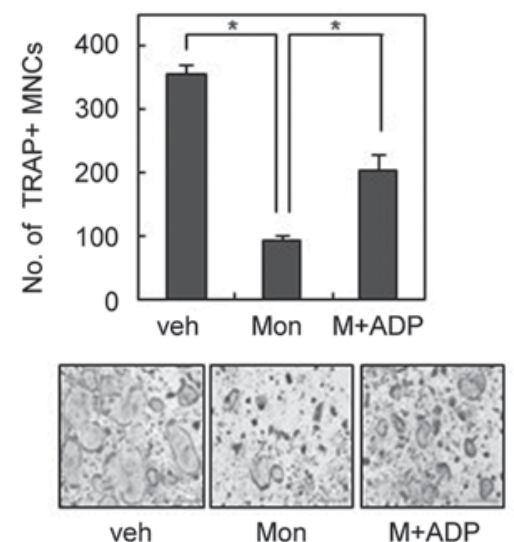

C

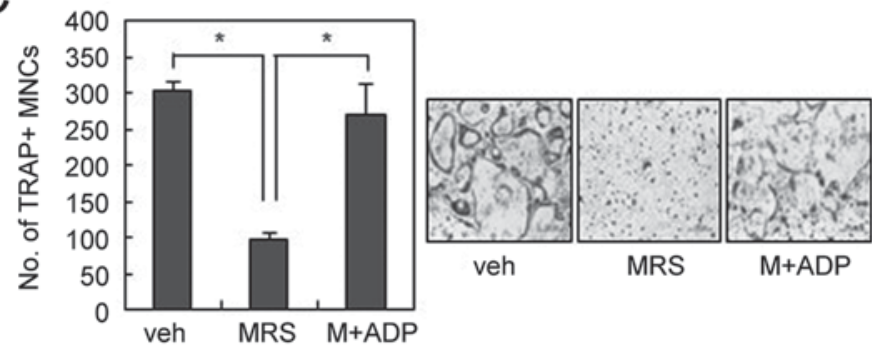

B

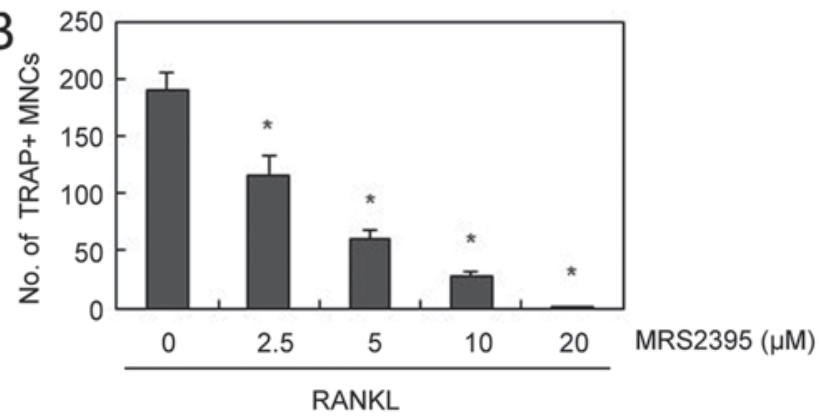

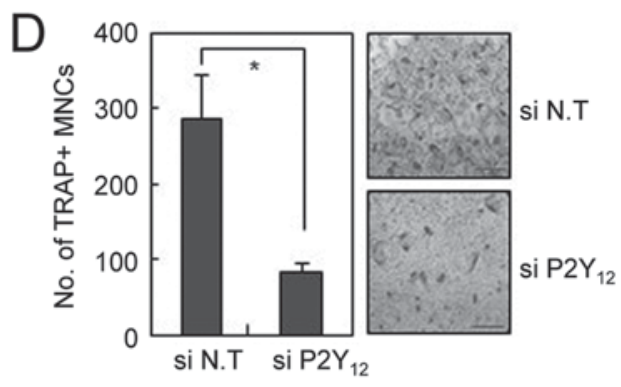
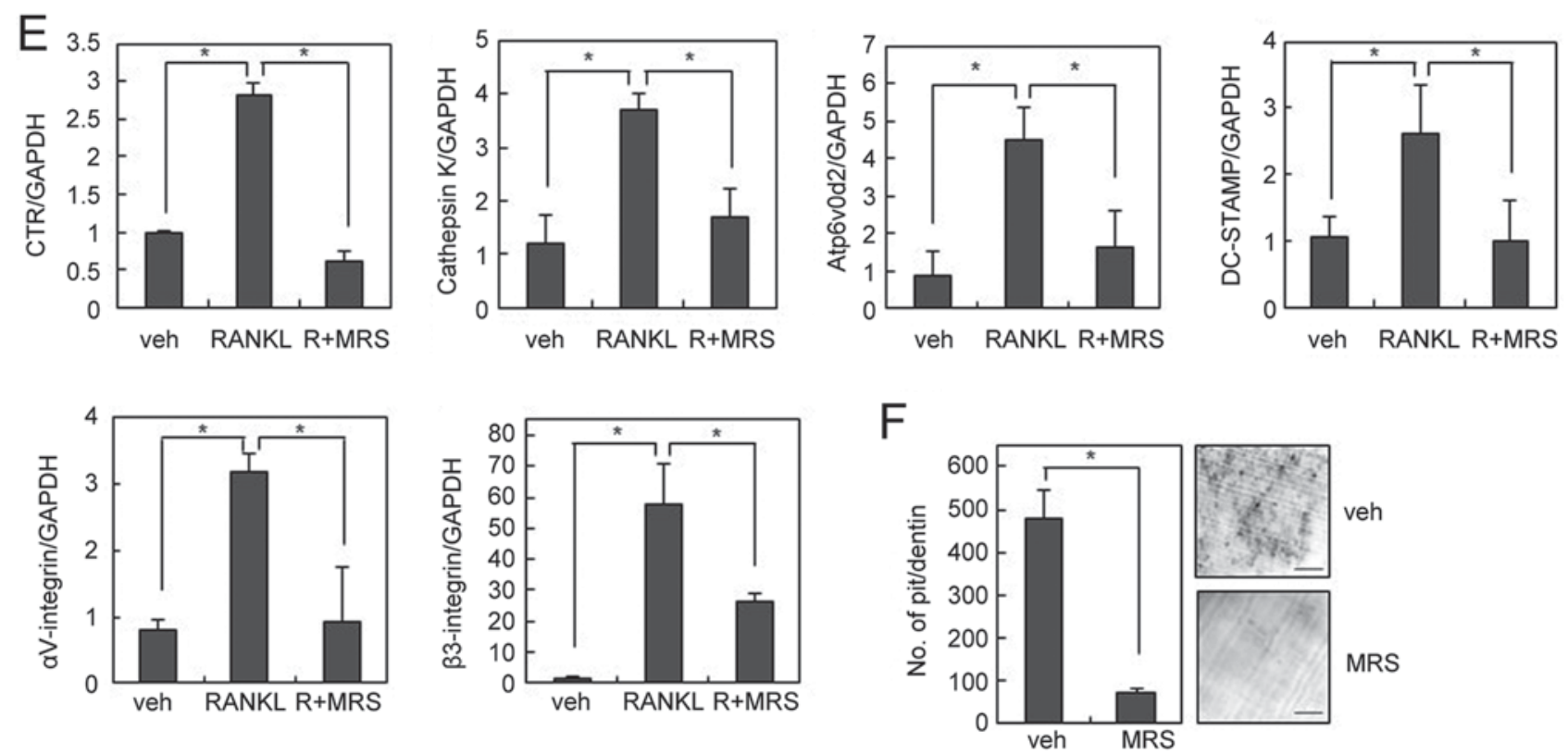

Figure 3. P2Y12 blockade inhibits RANKL-induced OC formation and bone resorption. (A) BMMs were cultured with montelukast (30 $\mu \mathrm{M})$ in the absence or presence of 2-MeS-ADP $(10 \mu \mathrm{M})$ during OC differentiation and stained for TRAP activity. (B) BMMs were cultured with M-CSF (30 ng/ml) and RANKL $(100 \mathrm{ng} / \mathrm{ml})$ in the absence or presence of MRS2395 for 4 days. (C) BMMs were cultured with MRS2395 $(20 \mu \mathrm{M})$ in the absence or presence of 2-MeS-ADP during OC differentiation. (D) BMMs were transfected with $40 \mathrm{nM}$ siRNA. The siRNA-transfected BMMs were cultured with M-CSF (30 ng/ml) and RANKL $(100 \mathrm{ng} / \mathrm{ml})$ for 3 days. (E) mRNA expression was determined by reverse transcription-quantitative polymerase chain reaction. (F) The resorbed pit numbers on dentine slices in the absence or presence of MRS2395 $(20 \mu \mathrm{M})$ were counted. Scale bars $=200 \mu \mathrm{m}$. Data are expressed as means \pm standard deviation from at least three independent experiments. " $\mathrm{P}<0.05$, as indicated. RANKL, receptor activator of nuclear factor- $\mathrm{KB}$ ligand; OCs, osteoclasts; BMM, bone marrow-derived macrophages; TRAP, tartrate resistant acid phosphatase; siRNA, small interfering RNA; veh, vehicle; R, RANKL; Mon, montelukast; MRS, MRS2395; M-CSF, macrophage colony-stimulating factor.

thickness (Ct.TH), and BMD compared with the vehicle group mice. By treating montelukast, unloading-induced cortical bone loss was suppressed (Fig. 6C). Taken together, these results suggest that the CysLTR1 antagonist prevented OVX- or hind limb unloading-induced bone destruction in vivo.

\section{Discussion}

Osteoporosis is a bone disease with low bone mass and bone fragility that increases the risk of bone fracture. It is characterized as progressive and excessive bone resorption caused by enhanced OC differentiation and/or activity $(1,2)$. RANKL is 

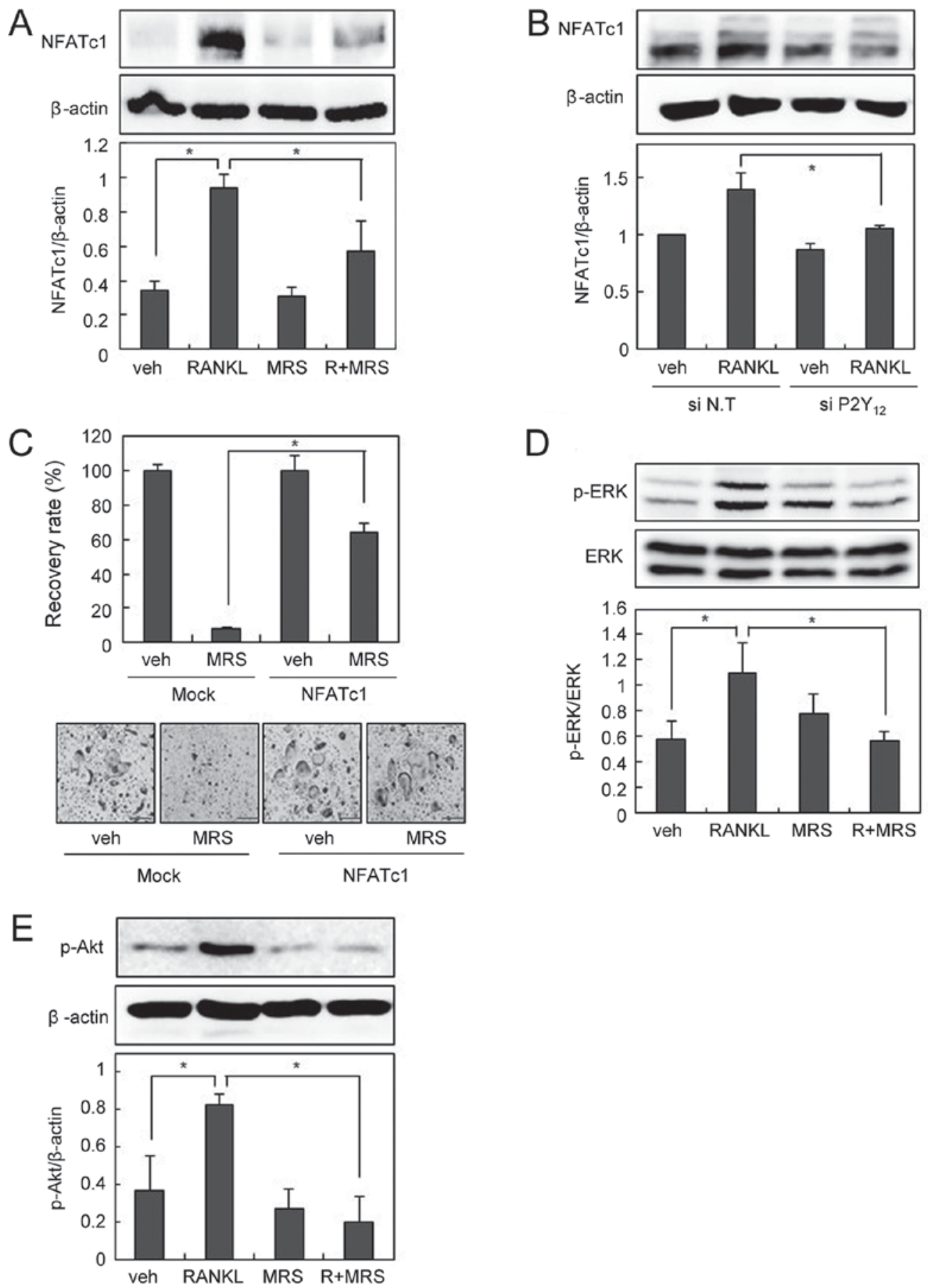

Figure 4. P2Y12 blockade suppresses RANKL-induced NFATc1 expression. (A) BMMs were pre-incubated in the absence or presence of MRS2395 (20 $\mu$ M) for $30 \mathrm{~min}$ and then cultured with or without RANKL $(200 \mathrm{ng} / \mathrm{ml})$ for $24 \mathrm{~h}$. Cell lysates were subjected to western blotting analysis. (B) The siRNA-transfected BMMs were cultured with RANKL ( $200 \mathrm{ng} / \mathrm{ml}$ ) for $24 \mathrm{~h}$. Cell lysates were then subjected to western blotting analysis. (C) Retrovirus-infected BMMs were cultured with RANKL $(100 \mathrm{ng} / \mathrm{ml})$ and M-CSF $(30 \mathrm{ng} / \mathrm{ml})$ in the presence or absence of MRS2395 $(20 \mu \mathrm{M})$ for 3 days. The recovery rate was defined as the percentage of osteoclast formation in the presence of MRS2395. BMMs were pre-incubated in the absence or presence of MRS2395 (20 $\mu$ M) for 30 min, and then treated with or without $200 \mathrm{ng} / \mathrm{ml}$ of RANKL for $15 \mathrm{~min}$. Cell lysates were then subjected to western blotting analysis for (D) p-ERK and ERK, and (E) p-Akt. Scale bars $=200 \mu \mathrm{m}$. Data are expressed as means \pm standard deviation from at least three independent experiments. "P $<0.05$, as indicated. RANKL, receptor activator of nuclear factor- $\mathrm{B}$ ligand; OCs, osteoclasts; BMM, bone marrow-derived macrophages; TRAP, tartrate resistant acid phosphatase; NFATc1, nuclear factor of activated T cells, cytoplasmic 1; p-, phosphorylated; ERK, extracellular signal-regulated kinase; Akt, protein kinase B; siRNA, small interfering RNA; veh, vehicle; R, RANKL; MRS, MRS2395.

a critical factor for OC differentiation, and excessive RANKL signaling results in enhanced OC formation and bone resorption $(3,4)$. As such, downregulation of RANKL expression or its downstream signals may be a valuable approach to the treatment of osteoporosis (2-4).
The CysLTs are a family of potent inflammatory lipid mediators derived from arachidonic acid through the 5-LO pathway (11). The CysLTR1 is a G-protein coupled receptor (GPCRs) and its interaction with CysLTs plays a central role in the pathophysiology of asthma and other 
A

veh

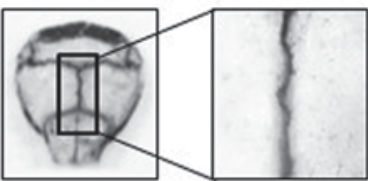

LPS

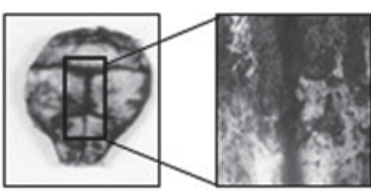

LPS+Mon

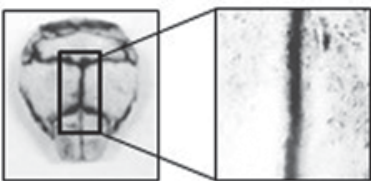

C
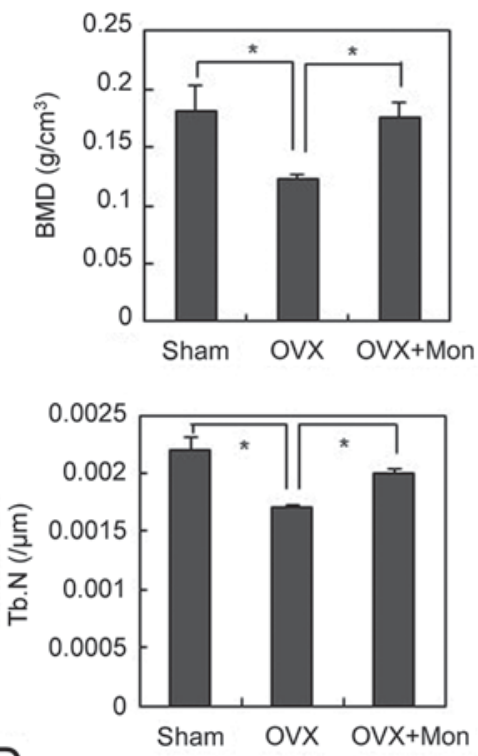

$\mathrm{D}$

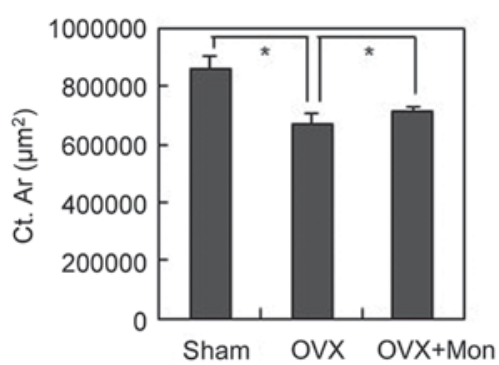

B

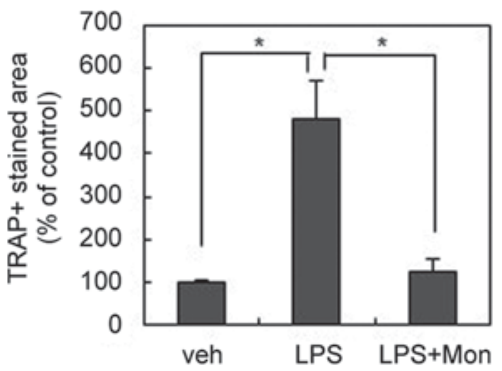

E

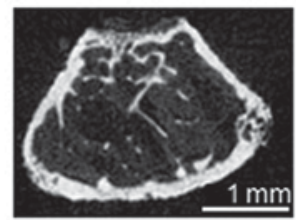

Sham
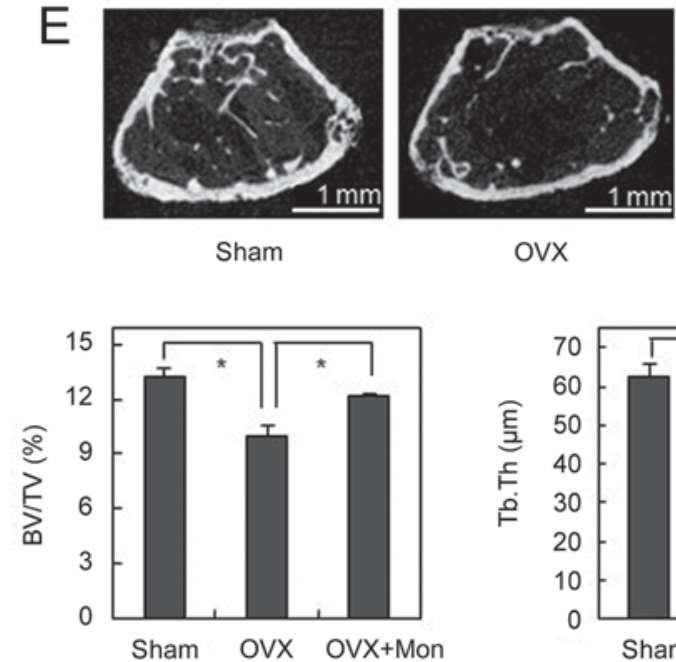

OVX

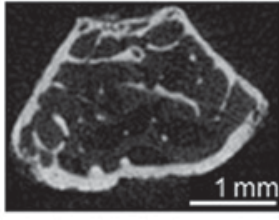

OVX+Mon
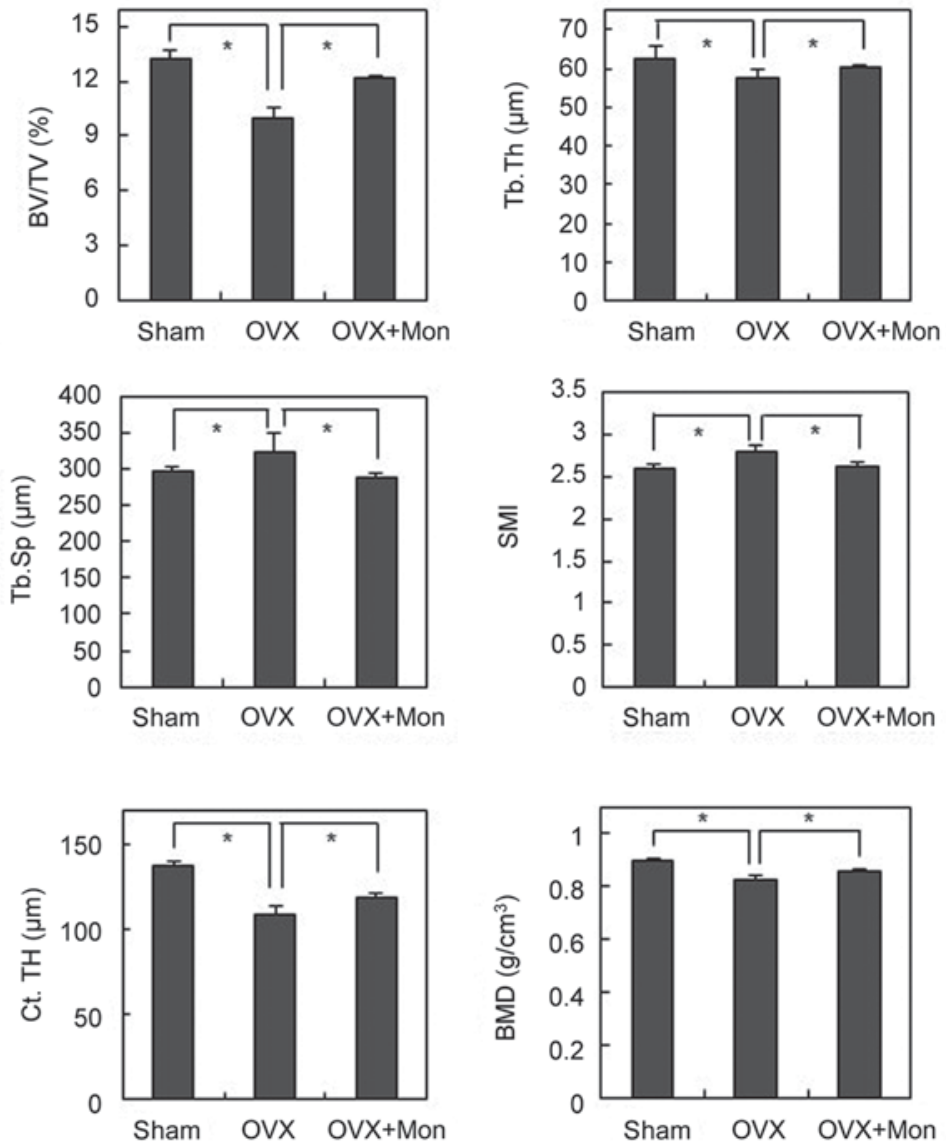

Figure 5. Montelukast inhibits OC formation and OVX-induced bone loss in vivo. (A) Calvariae of mice that received vehicle, LPS or LPS plus montelukast $(5 \mathrm{mg} / \mathrm{kg}$ ) were subjected to TRAP staining. (B) TRAP-positive stained areas in calvariae were quantified by using ImageJ. (C) The femurs of sham-operated, OVX and OVX plus montelukast mice were collected following 12 weeks. Various bone parameters were analyzed by micro-CT. (D) Two-dimensional micro-CT images of the distal metaphysis of the femur. (E) Micro-CT analysis for cortical bone. Scale bars $=1 \mathrm{~mm}$. Data are expressed as means \pm standard deviation from at least three independent experiments. " $\mathrm{P}<0.05$, as indicated. OCs, osteoclasts; TRAP, tartrate resistant acid phosphatase; LPS, lipopolysaccharide; OVX, ovariectomy; CT, computed tomography; BMD $\left(\mathrm{g} / \mathrm{cm}^{3}\right)$, bone mineral density; BV/TV (\%), bone volume per tissue volume; Tb.Th $(\mu \mathrm{m})$, trabecular thickness; Tb.N $(/ \mu \mathrm{m})$, trabecular number; Tb.Sp $(\mu \mathrm{m})$, trabecular separation; SMI, structural model index; Ct.Ar $\left(\mu \mathrm{m}^{2}\right)$, cortical area; $\mathrm{Ct} . \mathrm{TH}(\mu \mathrm{m}), \mathrm{cortical}$ thickness; BMD $\left(\mathrm{g} / \mathrm{cm}^{3}\right)$; veh, vehicle; Mon, montelukast.

inflammatory diseases (11-13). Several CysLTR1 antagonists have been developed to date and are currently in clinical practice $(13,21)$. Montelukast, probably due to its once daily dosing schedule, safety, and efficacy profile, is the most widely prescribed CysLTR1 antagonist worldwide. These agents are specifically used in the treatment of allergic rhinitis, exerciseand aspirin-induced asthma, and as add-on therapy for patients with asthma poorly controlled by inhaled corticosteroid (ICS) monotherapy or ICS in combination with long-acting $\beta 2$-agonists $(21,22)$. 
A

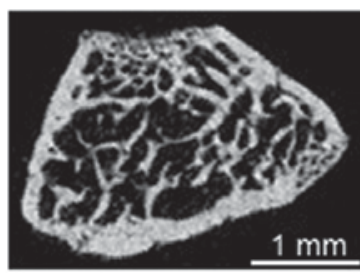

con

B
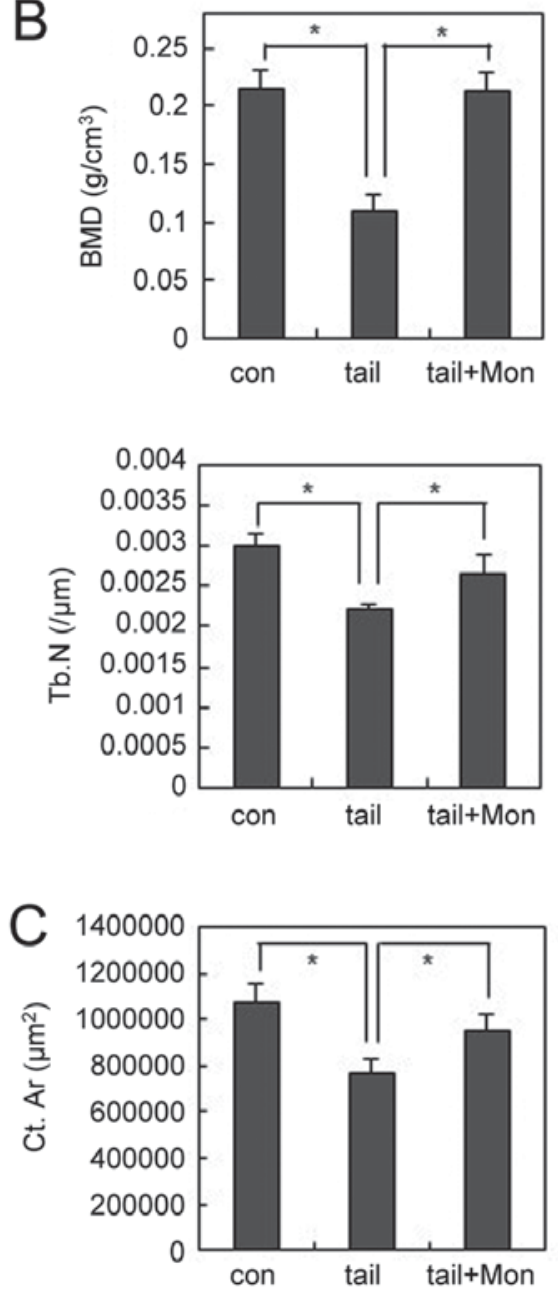

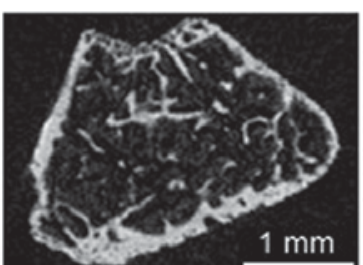

tail

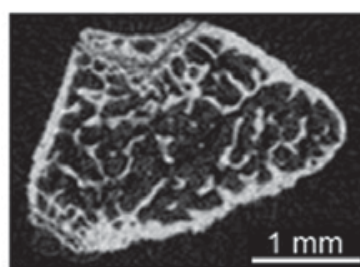

tail+Mon
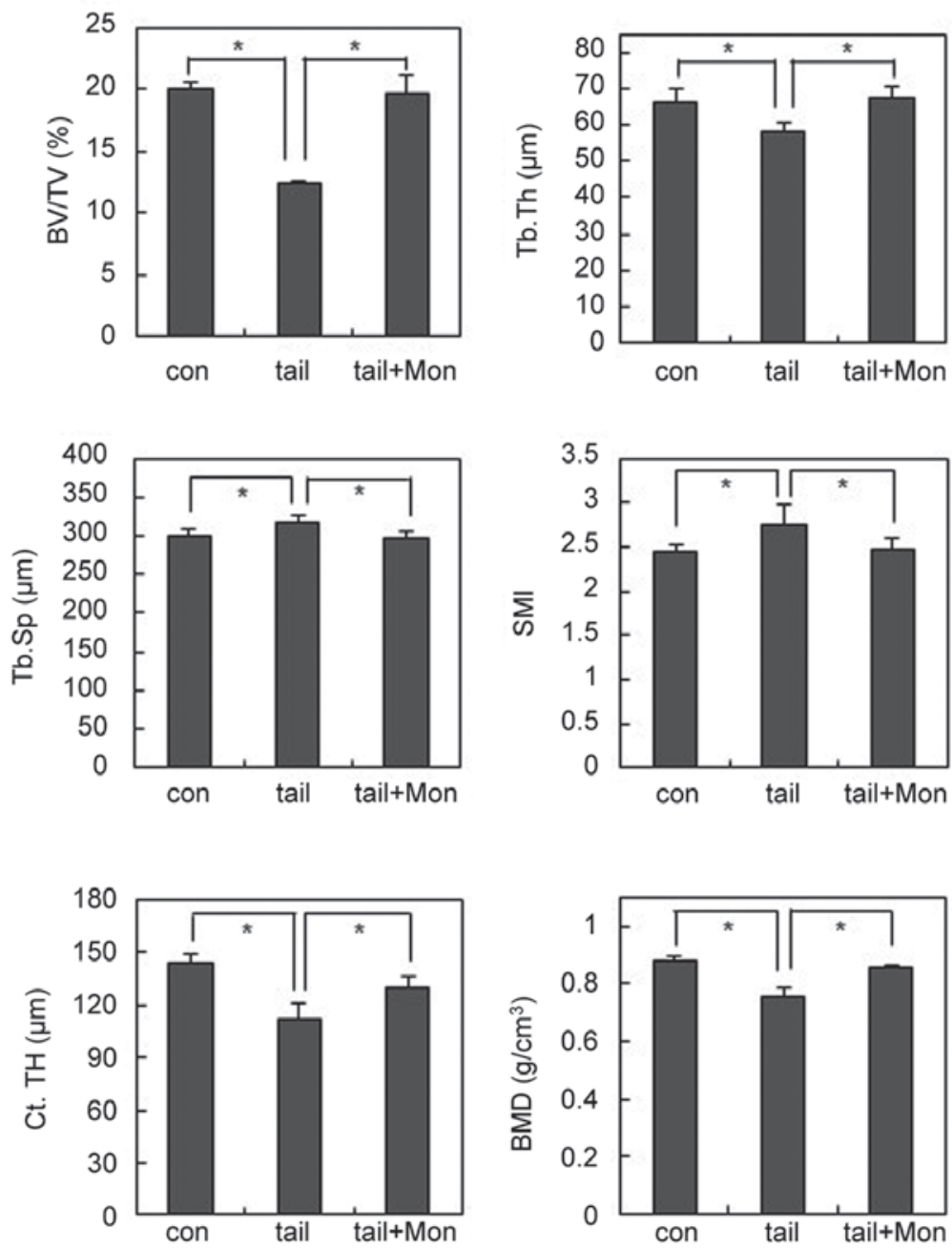

Figure 6. Montelukast prevents unloading-induced bone loss in vivo. The femurs of control, unloading, and unloading plus montelukast mice were collected following 2 weeks. (A) Two-dimensional micro-CT images of the distal metaphysis of the femurs. Scale bars=1 mm. (B) Various bone parameters of femurs were analyzed by micro-CT, including BMD $\left(\mathrm{g} / \mathrm{cm}^{3}\right)$, BV/TV $(\%)$, Tb.Th $(\mu \mathrm{m})$, Tb.N $(/ \mu \mathrm{m})$, Tb.Sp ( $\left.\mu \mathrm{m}\right)$ and SMI. (C) Micro-CT analysis for cortical bone. Data are expressed as means \pm standard deviation from at least three independent experiments. ${ }^{*} \mathrm{P}<0.05$, as indicated. $\mathrm{CT}$, computed tomography; $\mathrm{BMD}\left(\mathrm{g} / \mathrm{cm}^{3}\right)$, bone mineral density; BV/TV (\%), bone volume per tissue volume; Tb.Th $(\mu \mathrm{m})$, trabecular thickness; Tb.N (/ $\mu \mathrm{m})$, trabecular number; Tb.Sp ( $\mu \mathrm{m})$, trabecular separation; SMI, structural model index; Ct.Ar $\left(\mu \mathrm{m}^{2}\right)$, cortical area; Ct.TH $(\mu \mathrm{m})$, cortical thickness; BMD (g/cm $\left.{ }^{3}\right)$; con, control; tail, hind limb unloading; Mon, montelukast.

In this study, we showed that montelukast potently prevented RANKL-induced OC formation and bone loss in vivo. Montelukast dramatically downregulated RANKL-induced expression of NFATc1 in BMM culture. The forced expression of NFATc1 completely restored the inhibition of osteoclastogenesis by montelukast, suggesting that the inhibitory effect of montelukast is mediated at least partially through NFATc1.

NFATc1 activation requires activation of the MAPKs, Akt, and/or PLC $\gamma 2$ signaling (5-7). Our results further suggest that montelukast suppresses RANKL-induced NFATc1 expression at least in part by inhibiting the ERK, Akt, and PLC $\gamma 2$ pathways in BMMs. Upon RANKL stimulation, immunoreceptor tyrosine-based activation motif (ITAM)-bearing adaptors, Fc receptor common $\gamma$ subunit $(\mathrm{FcR} \gamma)$ and DNAX-activating protein 12 (DAP12), deliver co-stimulatory signals through activation of PLC $\gamma$. Activated PLC $\gamma$ generates inositol-1,4,5-triphosphate $\left(\mathrm{IP}_{3}\right)$, which mobilizes $\mathrm{Ca}^{2+}$ from the endoplasmic reticulum stores through inositol triphosphate receptors ( $\left.\mathrm{IP}_{3} \mathrm{Rs}\right)(23-26)$ and, subsequently, generates $\mathrm{Ca}^{2+}$ oscillation. This RANKL-induced $\mathrm{Ca}^{2+}$ oscillation activates CaMKIV, followed by CREB activation (9), which is critical for the activation of NFATc1 activation. Thus, PLC $\gamma 2 / \mathrm{Ca}^{2+} / \mathrm{CREB} / \mathrm{NFATc} 1$ signaling is an 
important pathway for OC differentiation and function in addition to MAPK and/or Akt pathways. Given that the interaction of CysLTs with CysLTR1 evokes $\mathrm{Ca}^{2+}$ oscillation (26,27), montelukast seems to suppress OC formation partly by attenuating the PLC $\gamma 2 / \mathrm{Ca}^{2+} / \mathrm{CREB} / \mathrm{NFATc} 1$ signaling pathway.

Recent evidence suggests that montelukast possesses a range of secondary activities independent of CysLTR1, including inhibition of 5-LO, histone acetyltransferase, cAMP phosphodiesterase, and interference with purinergic P2Y12 receptors (28). In particular, phylogenetic analysis of the P2Y12 and CysLTR1 indicated that CysLTR1 is closely related to P2Y12 (29). Furthermore, a computer model predicted that CysLTs act as surrogate ligands for the P2Y12 receptor (30), suggesting that CysLTR1 antagonists interact functionally with P2Y12 receptor signaling pathways. In this study, we demonstrated that the inhibitory effect of montelukast on osteoclastogenesis was efficiently restored by addition of P2Y12 agonist and by that of CysLT. Furthermore, P2Y12 antagonist inhibited osteoclast formation through signaling pathways similar to those induced by montelukast. Thus, we suggest that montelukast might exert its effect on OC formation by antagonizing the CysLTR1 and P2Y12 signaling.

Drug repositioning is progressively getting attention as a promising method for drug discovery. A repositioned compound with proven bioavailability and known safety profile presents many advantages such as an accelerated R\&D process, reduced development cost, and decreased failure rate due to safety (31). Montelukast is an FDA-approved anti-asthmatic drug used to treat asthma and allergic rhinitis. Herein, we show that montelukast inhibits osteoclastogenesis by suppressing the expression of NFATc1 via ERK, Akt, and PLC $\gamma 2$ pathways. Furthermore, montelukast prevented ovariectomy- or disuse-induced bone loss. Taken together, our data suggest that montelukast is a potential repositioned drug for treating bone diseases associated with excessive bone resorption.

\section{Acknowledgements}

Not applicable.

\section{Funding}

The present study was supported by grants from the Korean Health Technology R\&D Project, Ministry of Health \& Welfare, Republic of Korea (grant no. HI14C24470000) and from the National Research Foundation of Korea (NRF), funded by Ministry of Science, ICT and Future Planning (MSIP; grant nos. NRF-2014M1A3A3A02034917 and NRF-2016R1A2B4011636).

\section{Availability of data and materials}

The datasets used and/or analyzed during the current study are available from the corresponding author on reasonable request.

\section{Authors' contributions}

MY participated in research design. JHK conducted the experiments. JHK, HL, DSL and MY performed data analysis. MY wrote the manuscript.

\section{Ethics approval and consent to participate}

All experiments were performed in accordance with institutional guidelines approved by the Sookmyung Women's University Care and Use Committee.

\section{Consent for publication}

Not applicable.

\section{Competing interests}

The authors declare that they have no competing interests.

\section{References}

1. Zaidi M: Skeletal remodeling in health and disease. Nat Med 13: 791-801, 2007.

2. Rodan GA and Martin TJ: Therapeutic approaches to bone diseases. Science 289: 1508-1514, 2000.

3. Boyle WJ, Simonet WS and Lacey DL: Osteoclast differentiation and activation. Nature 423: 337-342, 2003.

4. Suda T, Takahashi N, Udagawa N, Jimi E, Gillespie MT and Martin TJ: Modulation of osteoclast differentiation and function by the new members of the tumor necrosis factor receptor and ligand families. Endocr Rev 20: 345-357, 1999.

5. Lee ZH and Kim HH: Signal transduction by receptor activator of nuclear factor kappa B in osteoclasts. Biochem Biophys Res Commun 305: 211-214, 2003.

6. Moon JB, Kim JH, Kim K, Youn BU, Ko A, Lee SY and Kim N: Akt induces osteoclast differentiation through regulating the GSK3 $\beta /$ NFATc1 signaling cascade. J Immunol 188: 163-169, 2012.

7. Faccio R and Cremasco V: PLCgamma2: Where bone and immune cells find their common ground. Ann N Y Acad Sci 1192: 124-130, 2010.

8. Takayanagi H, Kim S, Koga T, Nishina H, Isshiki M, Yoshida H, Saiura A, Isobe M, Yokochi T, Inoue J, et al: Induction and activation of the transcription factor NFATc1 (NFAT2) integrate RANKL signaling in terminal differentiation of osteoclasts. Dev Cell 3: 889-901, 2002.

9. Sato K, Suematsu A, Nakashima T, Takemoto-Kimura S, Aoki K, Morishita Y, Asahara H, Ohya K, Yamaguchi A, Takai T, et al: Regulation of osteoclast differentiation and function by the CaMK-CREB pathway. Nat Med 12: 1410-1416, 2006.

10. Negishi-Koga T and Takayanagi $\mathrm{H}$ : Ca2+-NFATc1 signaling is an essential axis of osteoclast differentiation. Immunol Rev 131: 241-256, 2009

11. Singh RK, Gupta S, Dastidar S and Ray A: Cysteinyl leukotrienes and their receptors: Molecular and functional characteristics. Pharmacology 85: 336-349, 2010.

12. Nayak A: A review of montelukast in the treatment of asthma and allergic rhinitis. Expert Opin Pharmacother 5: 679-686, 2004.

13. Theron AJ, Steel HC, Tintinger GR, Gravett CM, Anderson R and Feldman C: Cysteinyl leukotriene receptor-1 antagonists as modulators of innate immune cell function. J Immunol Res 2014: 608930, 2014.

14. Hikiji H, Takato T, Shimizu T and Ishii S: The roles of prostanoids, leukotrienes and platelet-activating factor in bone metabolism and disease. Prog Lipid Res 47: 107-126, 2008.

15. Livak KJ and Schmittgen TD: Analysis of relative gene expression data using real-time quantitative PCR and the 2(-Delta Delta C(T)) method. Method 25: 402-208, 2001.

16. Ishijima M, Rittling SR, Yamashita T, Tsuji K, Kurosawa H, Nifuji A, Denhardt DT and Noda M: Enhancement of osteoclastic bone resorption and suppression of osteoblastic bone formation in response to reduced mechanical stress do not occur in the absence of osteopontin. J Exp Med 193: 399-404, 2001.

17. Virchow JC and Bachert C: Efficacy and safety of montelukast in adults with asthma and allergic rhinitis. Respir Med 100: 1952-1959, 2006

18. Wagner EF and Karsenty G: Genetic control of skeletal development. Curr Opin Genet Dev 11: 527-532, 2001. 
19. Wada T, Nakashima T, Hiroshi $\mathrm{N}$ and Penninger JM: RANKL-RANK signaling in osteoclastogenesis and bone disease. Trends Mol Med 12: 17-25, 2006.

20. Ha H, Lee JH, Kim HN, Kim HM, Kwak HB, Lee S, Kim HH and Lee ZH: alpha-Lipoic acid inhibits inflammatory bone resorption by suppressing prostaglandin E2 synthesis. J Immunol 176: $111-117,2006$.

21. Riccioni G, Bucciarelli T, Mancini B, Di Ilio C and D'Orazio N: Antileukotriene drugs: Clinical application, effectiveness and safety. Curr Med Chem 14: 1966-1977, 2007.

22. Paggiaro P and Bacci E: Montelukast in asthma: A review of its efficacy and place in therapy. Ther Adv Chronic Dis 2: 47-58, 2011.

23. Kim N, Takami M, Rho J, Josien R and Choi Y: A novel member of the leukocyte receptor complex regulates osteoclast differentiation. J Exp Med 195: 201-209, 2002.

24. Koga $\mathrm{T}$, Inui M, Inoue K, Kim S, Suematsu A, Kobayashi E, Iwata T, Ohnishi H, Matozaki $\mathrm{T}$ and Kodama $\mathrm{T}$, et al: Costimulatory signals mediated by the ITAM motif cooperate with RANKL for bone homeostasis. Nature 428: 758-763, 2004.

25. Ferron M, Boudiffa M, Arsenault M, Rached M, Pata M Giroux S, Elfassihi L, kisseleva M, Majerus PW, Rousseau F and Vacher J: Inositol polyphosphate 4-phosphatase B as a regulator of bone mass in mice and humans. Cell Metab 14: 466-477, 2011.
26. Kim H, Kim T, Jeong BC, Cho IT, Han D, Takegahara N, Negishi-Koga T, Takayanagi H, Lee JH, Sul JY, et al: Tmem64 modulates calcium signaling during RANKL-mediated osteoclast differentiation. Cell Metab 17: 249-260, 2013.

27. Di Capite J, Shirley A, Nelson C, Bates G and Parekh AB Intercellular $\mathrm{Ca} 2+$ wave propagation involving positive feedback between CRAC channels and cysteinyl leukotrienes. FASEB J 3: 894-905, 2009.

28. Tintinger GR, Feldman C, Theron AJ and Anderson R: Montelukast: More than a cysteinyl leukotriene receptor antagonist? Sci World J 10: 2403-2413, 2010.

29. Fredriksson R, Lagerstrom MC, Lundin LG and Schioth HB: The G-protein-coupled receptors in the human genome form five main families. Phylogenetic analysis, paralogon groups and fingerprints. Mol Pharmacol 63: 1256-1272, 2003.

30. Nonaka Y, Hiramoto T and Fujita N: Identification of endogenous surrogate ligands for human P2Y12 receptors by in silico and in vitro methods. Biochem Biophys Res Commun 337: 281-288, 2005.

31. Finsterer $\mathrm{J}$ and Frank M: Repurposed drugs in metabolic disorders. Curr Top Med Chem 13: 2386-2394, 2013. 